\title{
ENHANCED TARGET TRACKING BASED ON MEAN SHIFT ALGORITHM FOR SATELLITE IMAGERY
}

\author{
Sarabjit Kaur ${ }^{1}$, Sukhjinder Kaur ${ }^{2}$ \\ ${ }^{1}$ M.Tech Scholar, ${ }^{2}$ Assistant Professor, Department of Electronics \& Communication Engineering, Sri Sukhmani Institute \\ of Engineering \& Technology, Derabassi (Punjab), India, techsarab@gmail.com, Sukhjinder.253@gmail.com
}

\begin{abstract}
Target tracking in high resolution satellite images is challenging task for computer vision field. In this paper we have proposed a mean shift algorithm based enhanced target tracking system for high resolution satellite imagery. In proposed tracking algorithm, Target modeling is done using spectral features of target object i.e. Mean \& Energy density function. Feature Vector space with minimum Euclidean Distance is used for predicting next possible position of target object in consecutive frames. Proposed tracking algorithm has been tested using two high resolution databases i.e. Harbor \& Airport region database acquired by WorldView-2 satellite at different times. Recall, Precision \& $F_{1}$ score etc. performance parameters are also calculated for showing the tracking ability of the proposed method in real-time applications and are compared with the results of Regional Operator Design based tracking algorithm proposed in [1]. The results show that our proposed method gives relatively better performance than the other tracking algorithms used in satellite imagery.
\end{abstract}

Keywords- Target tracking, Mean shift algorithm, Energy density function, Feature Vector Space, Frame

\section{INTRODUCTION}

High resolution satellite images are often used for Target tracking in real time applications such as surveillance and monitoring, smart rooms, human tracking in satellite frames etc. But doing this with high accuracy is an exigent job in computer vision field. So, whole liability lies on the robustness of the target tracking algorithm. Various methods for target tracking have been proposed and differ in context of which type of object representation, Image features \& target modeling is suitable for tracking purpose? The answers to these questions depend on the context/environment in which the tracking is performed and the end use for which the tracking information is being required. A large number of tracking methods have been proposed which attempt to answer these questions for a variety of scenarios [2]. Proposed by Dorin commaniciu et al [3] in object tracking field, Iterative Mean shift algorithm for object tracking is one of the strong contender among these algorithms, which provides fast \& robust performance for target tracking. It is a non-parametric mode-seeking, feature space analysis method with low computational complexity. Mean Shift algorithm is type of forward tracking which tracks by minimizing a distance between two probability density functions represented by a reference and candidate histogram [4]. Mean shift object tracking method can be used it in its various forms e.g. CAMShift, ABCshift etc. depending upon accuracy \& applications.
Lingfei Meng [1] has proposed a novel regional operator design based object tracking algorithm for target matching in satellite imagery. This algorithm uses both spectral \& spatial features for target modeling. C. Carrano implemented an ultrascale capable multiple-vehicle tracking algorithm for overhead persistent surveillance imagery which relies on the mover map, path dynamics, and image features to perform tracking [6]. Kerekes et al evaluated the feasibility for particular objects of interest to be located and tracked in sequential frames of hyperspectral imagery through the use of their potentially unique spectral reflectance characteristics and then using that information to find the same vehicle in a subsequent image [7].

In this paper we are aimed at using modified form of mean shift algorithm for target tracking in high resolution WV-2 satellite imagery. By making tracking algorithm proposed in [1] as a base for our research we have showed that employing mean shift algorithm for target object tracking in satellite imagery gives far better results with less computational complexity and tracks the target relatively faster than other methods.

\section{PROPOSED ENHANCED TARGET TRACKING}

\section{SYSTEM BASED ON MEAN-SHIFT ALGORITHM}

Mean shift is a non-parametric density gradient estimator. It is employed to derive the object candidate that is the most similar to a given model while predicting the next object 
location. In other words, it starts from the position of the model in the current frame and then searches in the model's neighborhood in next frame, followed by finding best candidate by maximizing a similarity function. Finally, repeats the same process in the next pair of frames [10].

The proposed enhanced target tracking system based on mean shift algorithm, tracks the target object as illustrated in fig- 1 . Basically it consists of two main steps i.e. Target Modeling \& Target Matching.

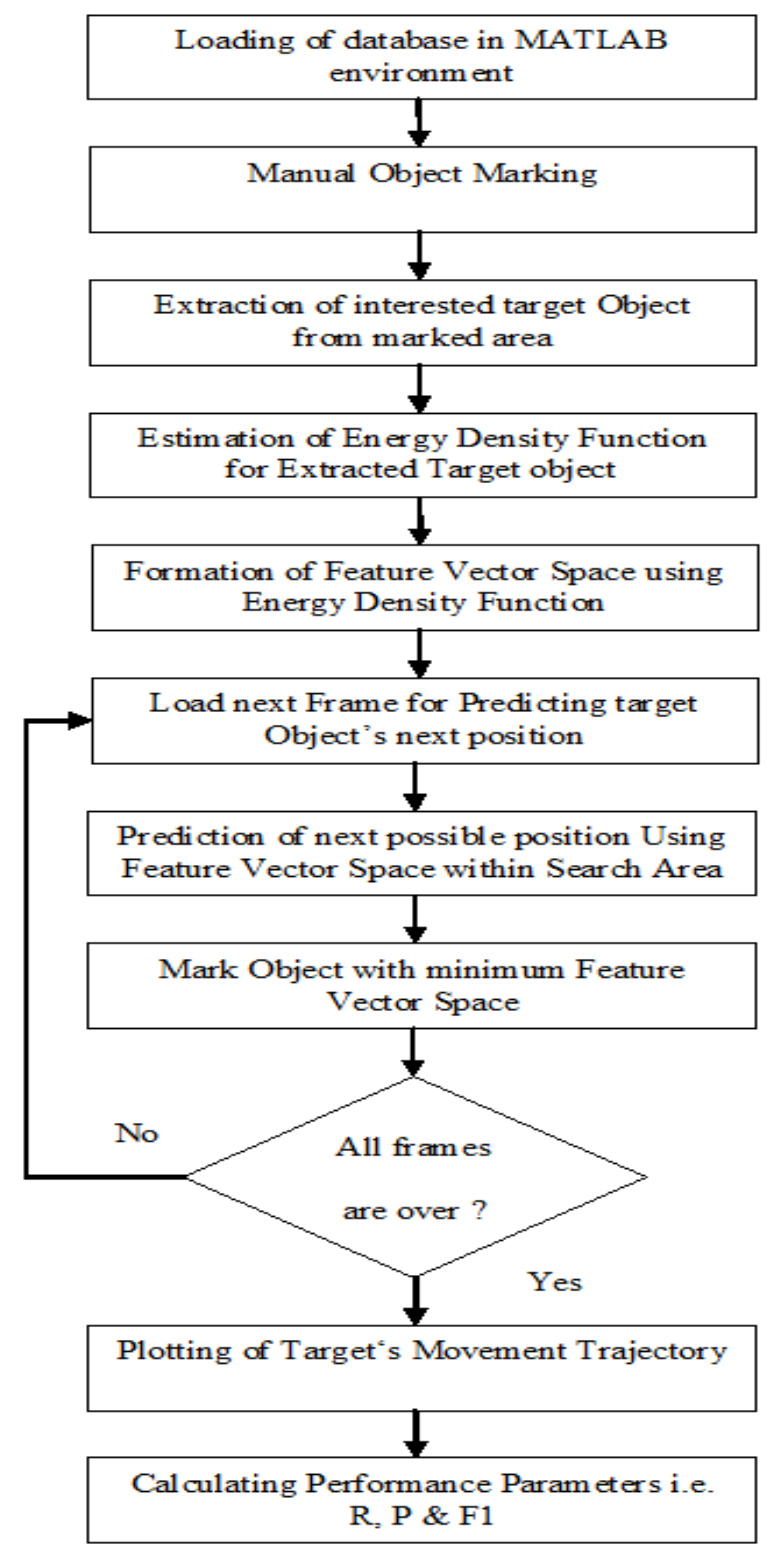

Fig -1: Flow chart of proposed method for target tracking

\subsection{Target Modeling}

The Target is defined as the interested object to be tracked [1] $\&$ Target model is a representation of chosen interested object in a current frame. The reference target in the current frame of used database is represented by a manually selected user defined area $\mathrm{K}$ as shown in fig 2.

Main Frame J

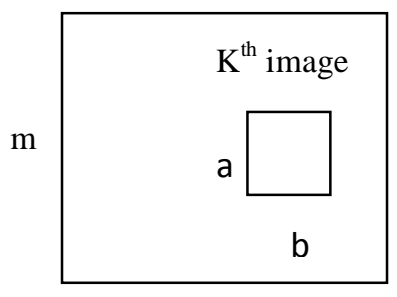

$\mathrm{n}$

Fig -2: A manually selected square area ' $\mathrm{K}$ ' from current main Frame J

The interested target is then extracted from this selected area for feature extraction purpose. The reference target is modeled by extracting its Spectral reflectance Characteristics. The Spectral Characteristic of target is defined as its probability density function (PDF). In our method Mean \& Energy density function (EDF) of reference target is used for PDF Estimation. Mean \& EDF of reference target can be determined as follows:

$\mathrm{Z}_{\mathrm{R}}=0$

$$
\mathrm{Z}_{\mathrm{R}}=\sum_{\mathrm{i}=1}^{\mathrm{i}=\mathrm{a}} \sum_{\mathrm{j}=1}^{\mathrm{j}=\mathrm{b}} \mathrm{Z}_{\mathrm{R}}+\mathrm{K}(\mathrm{i}, \mathrm{j}, 1) ;
$$

$\mathrm{Z}_{\mathrm{G}}=0$

$$
\mathrm{Z}_{\mathrm{G}}=\sum_{\mathrm{i}=1}^{\mathrm{i}=\mathrm{a}} \sum_{\mathrm{j}=1}^{\mathrm{j}=\mathrm{b}} \mathrm{Z}_{\mathrm{G}}+\mathrm{K}(\mathrm{i}, \mathrm{j}, 2) ;
$$

$Z_{B}=0$

$$
Z_{B}=\sum_{i=1}^{i=a} \sum_{j=1}^{j=b} Z_{B}+K(i, j, 3)
$$

Where $\mathrm{Z}_{\mathrm{R}}, \mathrm{Z}_{\mathrm{G}}$ \& $\mathrm{Z}_{\mathrm{B}}$ represents Red, Green \& blue components of reference target object.

$\mathrm{K}=$ user selected area in current frame having dimensions $\mathrm{a} \times \mathrm{b}$. 
Now,

$$
Y=\sum_{i=1}^{i=a} \sum_{j=1}^{i=b}\left\{\begin{array}{cc}
Y+1, & \text { if } K(i, j, 1)>0|K(i, j, 2)>0| K(i, j, 3)>0 \\
Y+0, & \text { otherwise }
\end{array}\right.
$$

Where $\mathrm{Y}=$ Total no. of effective pixels in extracted target Object.

So Energy density function can be determined as:

$$
\begin{gathered}
\mathrm{Z}_{\mathrm{R}}=\frac{\mathrm{Z}_{\mathrm{R}}}{\mathrm{Y}} \\
\mathrm{Z}_{\mathrm{G}}=\frac{\mathrm{Z}_{\mathrm{G}}}{\mathrm{Y}} \\
\mathrm{Z}_{\mathrm{B}}=\frac{\mathrm{Z}_{\mathrm{B}}}{\mathrm{Y}}
\end{gathered}
$$

\subsection{Target Matching}

For Target matching purpose, target model estimated in section 2.1 is used for finding similar target candidates within a search area in the next frame. Search Area is a predefined area within the next frame where target has a high probability to move. Starting with the position of the Extracted target model in the current frame, the best target candidate is found by looking for a candidate with a minimum Euclidean distance within the model's neighborhood (search area) in next frame. The same process is repeated in the next pair of frames. A Feature vector Space (FVS) is formed by calculating the Euclidean distance between reference target model pixels \& each pixel within the Search area in next frame and used for predicting next possible position of interested target in successive frames. Feature vector space is basically a feature table having set of all possible position vectors representing target candidates in the next frame.

\section{Pseudo code for FVS formation is as follows:}

Let $\mathrm{Z}=$ total no. of frames available for tracking $\mathrm{I}_{\mathrm{k}}=$ Specified Search Area or a search window of size $\mathrm{x} \times \mathrm{y}$ within which Tracking algorithm will find the next movement position of Target object.

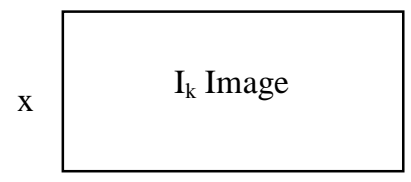

$\mathrm{y}$

for $\mathrm{k}=1$ to $\mathrm{Z}$

for $\mathrm{i}=1$ to $\mathrm{i}=\mathrm{x}$

for $\mathrm{j}=1$ to $\mathrm{j}=\mathrm{y}$

$\operatorname{FVS}(i, j)=\left|I_{K}(i . j, 1)-Z_{R}\right|+\left|I_{K}(i . j, 2)-Z_{G}\right|+\left|I_{K}(i . j, 3)-Z_{B}\right|(8)$ end loop

end loop

end loop

The position of the target candidate (any position vector in the search Area), with Minimum Euclidean Distance in the Feature vector table, will be the next possible position of the target in the successive frame. After estimating the possible positions of reference target in all the frames its movement trajectory is formed and compared with its manually identified ground truth trajectory for further performances evaluation.

\section{IMPLEMENTATION AND RESULTS}

The Proposed method for target tracking in satellite imagery has been evaluated using two databases harbor region \& airport region shown in fig $3 \& 4$ respectively. Experimental results using these databases have been obtained within MATLAB environment. Both databases consist of subsets of five frames, which are World- View-2 satellite image sequences provided by digital Globe (online) [13]. To make the methodology more understandable, some intermediate results for tracking of ship target in harbor region are shown in fig 5 which shows the steps like target modeling, target matching \& trajectory plotting.

Our aim was to track three targets i.e. ship target in harbor region database as shown in fig 6(a) \& aircraft-1\& aircraft-2 in airport region database as shown in fig 7(a) \& 8(a) respectively. Ground truth (GT) trajectory for these targets has been recognized manually per pixel in all the respective frames. Tracking performance evaluation of proposed method is done by calculating sensitivity (Recall), specificity, accuracy \& PPV like performance parameters, based on the number of true positives (TP), false positives (FP), and false negatives (FN) on per pixel basis as given in Table 1 . Table 2 shows comparative tracking performance of Regional Operator Design based tracking algorithm [1] \& our proposed tracking method for three selected targets. This performance comparison is also shown graphically in figure 9.

First ship target has been tracked successfully in harbor region database \& its movement trajectory has been estimated in fig 6(b). Then two aircrafts in airport region database have been tracked with their movement trajectory estimation as shown in fig 7(b) \& 8(b). The size of three targets and the size of Search area used to find them in next frames, is given in Table 2. 
Table -1: Tracking performance evaluating parameters \& their calculation [11][12]

\begin{tabular}{|l|l|}
\hline Measure & Description \\
\hline Recall or Sensitivity & $\mathrm{TP} /(\mathrm{TP}+\mathrm{FN})$ \\
\hline $\begin{array}{l}\text { Precision or Positive } \\
\text { Predictive Value }\end{array}$ & $\mathrm{TP} /(\mathrm{TP}+\mathrm{FP})$ \\
\hline Accuracy $($ Acc) & $(\mathrm{TP}+\mathrm{TN}) /(\mathrm{TP}+\mathrm{FP}+\mathrm{TN}+\mathrm{FN})$ \\
\hline Specificity $(\mathrm{SP})$ & $\mathrm{TN} /(\mathrm{TP}+\mathrm{FN})$ \\
\hline F1-Score & $2 *(\mathrm{R} * \mathrm{P}) /(\mathrm{R}+\mathrm{P})$ \\
\hline $\begin{array}{l}\text { False discovery rate } \\
\text { (FDR) }\end{array}$ & $\mathrm{FP} /(\mathrm{FP}+\mathrm{TP})$ \\
\hline
\end{tabular}

Movement trajectories of all the three tracked targets show high resemblance to manually identified GT trajectory. Hence, experimental results show the high tracking accuracy \& robustness of our proposed method.

Table -2: Feature vector space parameters

\begin{tabular}{|l|l|l|}
\hline Target & $\begin{array}{l}\text { Actual size } \\
\text { (Pixel) }\end{array}$ & $\begin{array}{l}\text { Search Area } \\
\text { (pixel x pixel ) }\end{array}$ \\
\hline Ship & 26 & $120 \times 20$ \\
\hline Aircraft-1 & 66 & $120 \times 20$ \\
\hline Aircraft-2 & 79 & $160 \times 20$ \\
\hline
\end{tabular}

\section{CONCLUSION}

We have proposed an enhanced \& robust, mean shift based target tracking system for satellite imagery which is able to track interested target with a high accuracy \& outperforms other tracking algorithms. Using only spectral features for target modeling \& Feature vector space with Euclidean distance as a similarity measure for target matching provides good performance. As shown through experimental results, three targets have been tracked successfully using proposed method with good tracking performance. Sensitivity (Recall), Precision \& F1 score etc. parameters are also calculated for showing better tracking performance of our algorithm than regional operator design based tracking algorithm. Future scope is to extend proposed enhanced mean shift method for target velocity estimation \& tracking of objects with small geographical area in complex environment with change in shape \& occlusion problem.

\section{REFERENCES}

[1] Lingfei Meng, Student Member, IEEE, and John P. Kerekes, Senior Member, IEEE "Object Tracking Using High Resolution Satellite Imagery" IEEE February 2012.

[2] A. Yilmaz, O. Javed, and M. Shah, "Object tracking: A survey,” ACM Comput. Surv., vol. 38, Dec. 2006.
[3] Comaniciu D., Ramesh V., Meer P.: 'Kernel-Based Object Tracking', IEEE Trans. Pattern Anal. Machine Intell. 2003, 25, (2), pp. 564-577.

[4] Tomas Vojir, Jana Noskova,Jiri Matas "Robust ScaleAdaptive Mean-Shift for Tracking" Springer-verlag berlin Heidelberg 2013 .

[5] Comaniciu D, Ramesh V, and Meer P, "Real-Time tracking of non-rigid objects using mean shift," In: Proc. of the IEEE Conf. on Computer Vision and Pattern Recognition (CVPR), pp.142-149, 2000.

[6] C. Carrano, "Ultra-scale vehicle tracking in low spatial resolution and low frame-rate overhead video," in Proc. SPIE, vol. 7445, 744504,2009.

[7] J. Kerekes, M. Muldowney, K. Strackerhan, L. Smith, and B. Leahy, "Vehicle tracking with multi-temporal hyperspectral imagery," in Proc. SPIE, vol. 6233, 62330C, 2006.

[8] Snekha, Chetna Sachdeva, Rajesh Birok "Real Time Object Tracking Using Different Mean Shift Techniques-a Review" International Journal of Soft Computing and Engineering (IJSCE) ISSN: 2231-2307, Volume-3, Issue-3, July 2013

[9] Zhi-Qiang Wen, Zi-xing Cai "Mean Shift Algorithm and its Application in Tracking of Objects" Proceedings of the Fifth International Conference on Machine Learning and Cybernetics, Dalian, 13-16 August 2006

[10] Zhu Chaoyang "Video Object Tracking using SIFT and Mean Shift" Chalmers University of Technology Göteborg, Sweden, 2011

[11] Muhammad Moazam Fraz, Paolo Remagnino, Andreas Hoppe, Bunyarit Uyyanonvara, Alicja R. Rudnicka, Christopher G. Owen, and Sarah A. Barman "An Ensemble Classification-Based Approach Applied to Retinal Blood Vessel Segmentation" IEEE Septemeber 2012.

[12] C. J. Van Rijsbergen, Information Retrieval, 2nd ed. Newton, MA: Butterworth-Heinemann, 1979.

[13] IEEE DigitalGlobe 2011 Data Fusion Contest. [Online]. Available:http://www.grss-ieee.org/2011-ieee digitalglobe-data-fusion-contest/

\section{BIOGRAPHIES:}

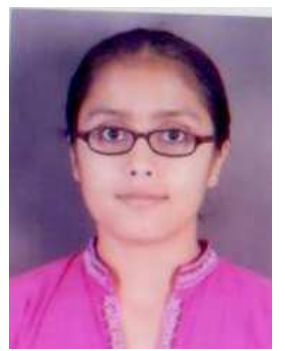

Sarabjit Kaur received her B.E degree in Electrical \& Electronics Communication Engineering from Chandigarh College of Engineering \& Technology, Chandigarh (U.T.) in 2009. Currently she is pursuing her Masters of Technology degree in Electronics \& Communication Engineering from SSIET, Derebassi (Punjab), a Regional centre of Punjab Technical University Jalandhar (Punjab). Her areas of interest are Digital image processing, Satellite Communication, Radar Engineering. 
Ms. Sukhjinder Kaur received her AMIE degree in Electronics \& Communication Engineering from the Institution of Engineers, Kolkata \& her M.Tech degree in Electronics \& Communication Engineering from Punjab Technical University, Jalandhar (Punjab). She is currently working as an Assistant Professor in the Department of
Electronics \& Communication Engineering at Sri Sukhmani Institute of Engineering and Technology, Derabassi (Punjab). Her research interests are Digital image processing \& Digital Signal Processing.

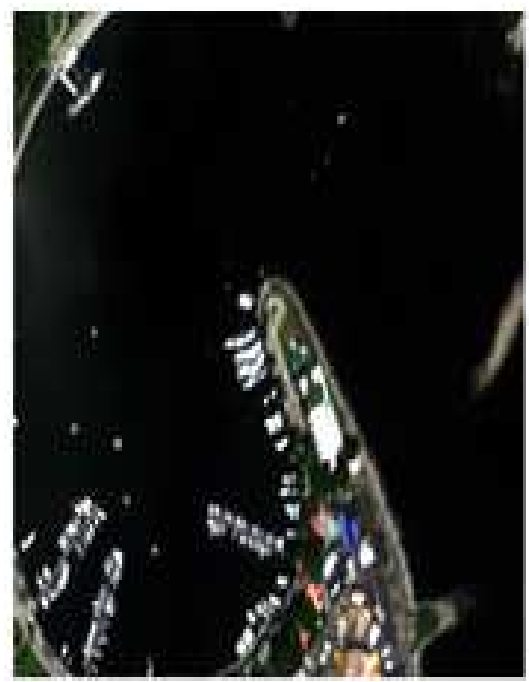

(ii)

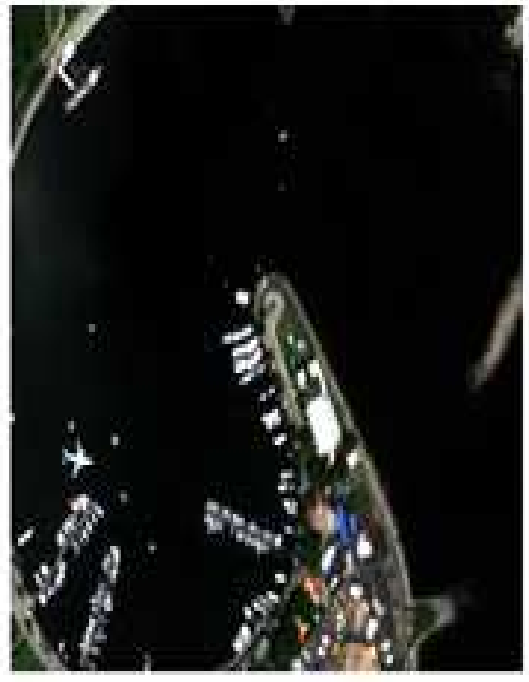

(b)

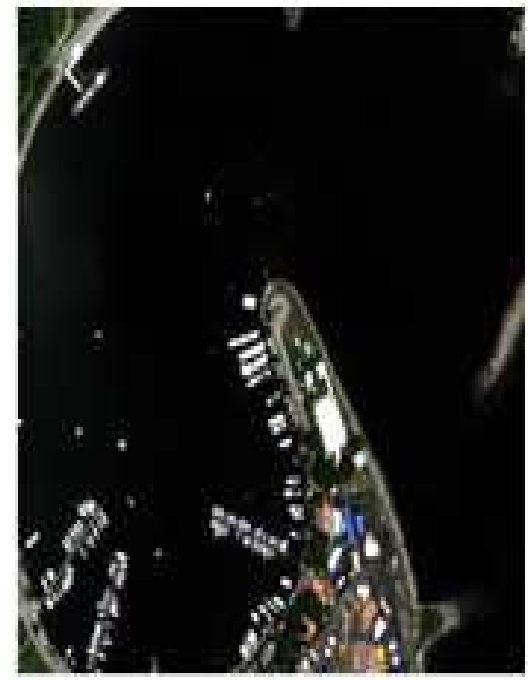

(a)

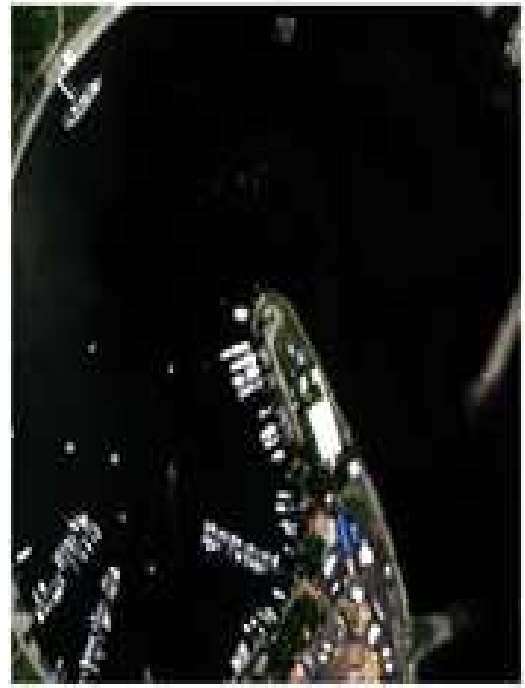

(d)

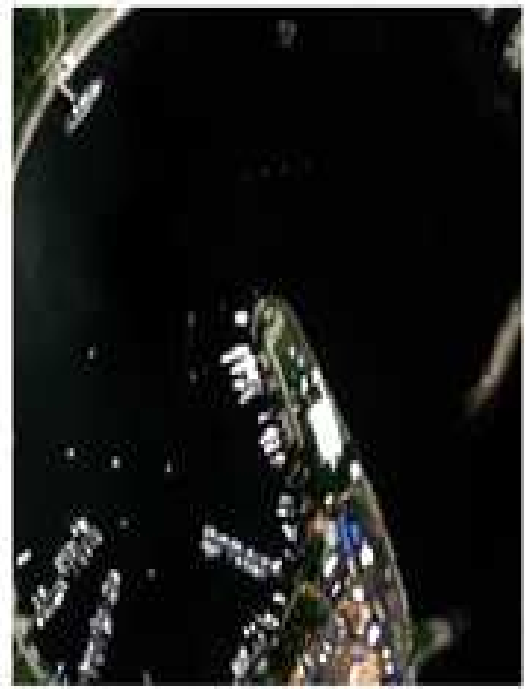

(c)

Fig -3: Harbor region database consists of subset of five frames collected at different times (a) 13:09:23, (b)13:09:54, (c) 13:10:46, (d) 13:12:00, and (e) 13:12:41. All times are local. 


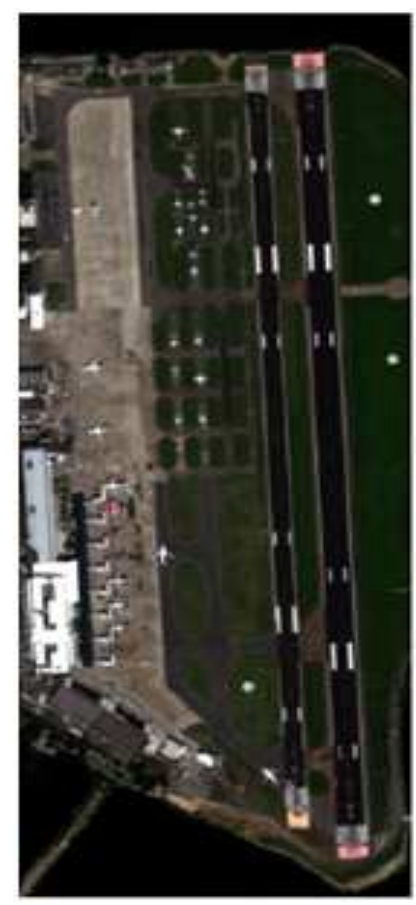

(a)

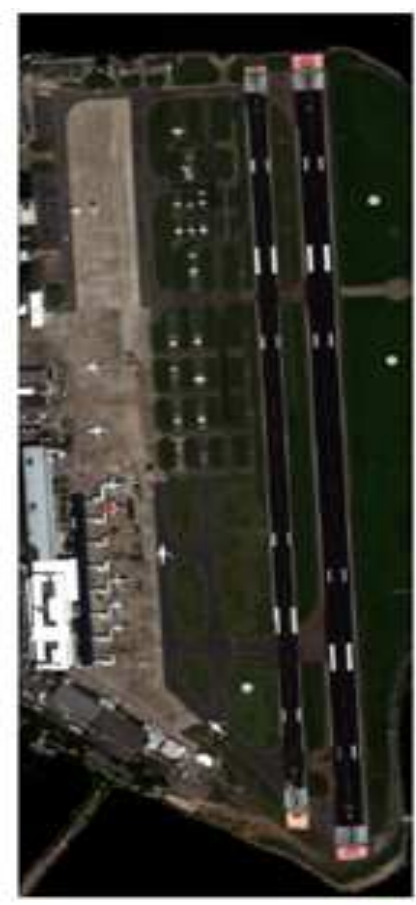

(b)

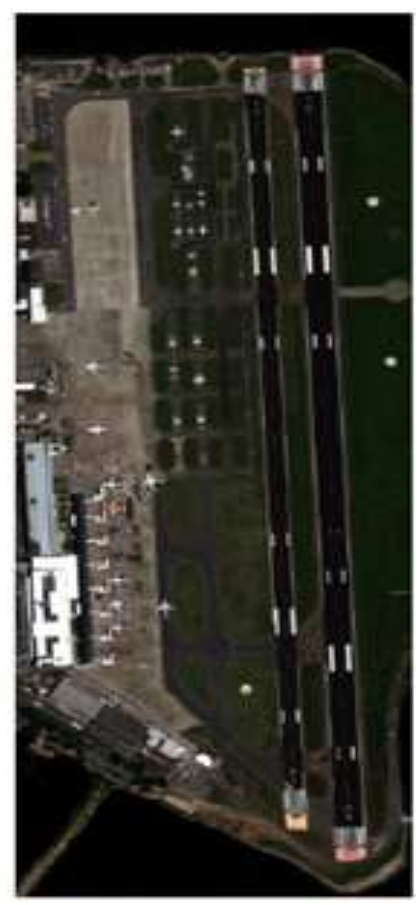

(c)

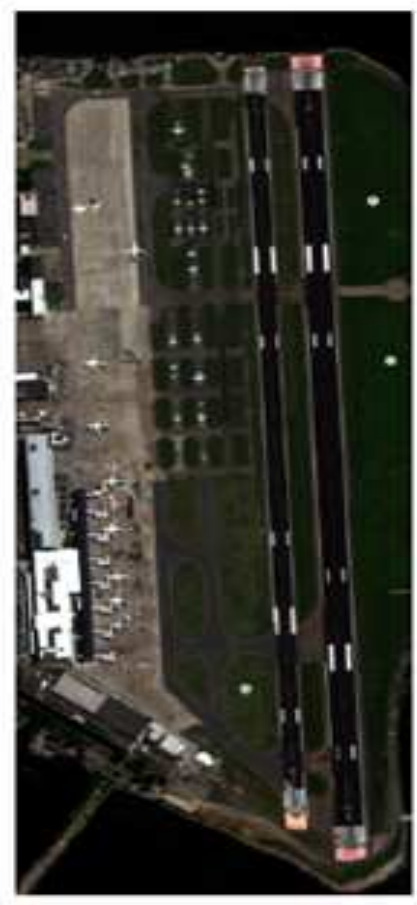

(d)

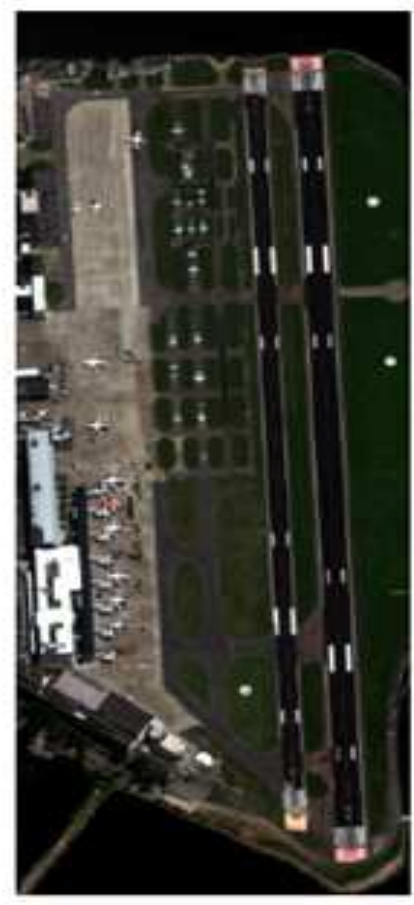

(e)

Fig -4: An airport region database consists of subset of five frames collected at different times (a) 13:09:23, (b) 13:09:54, (c) 13:10:46, (d) 13:12:00, and (e) 13:12:41. All times are local 


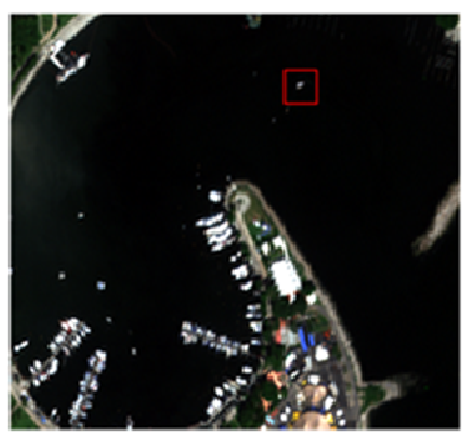

(a) Harbor region RGB image with ship target within red outline

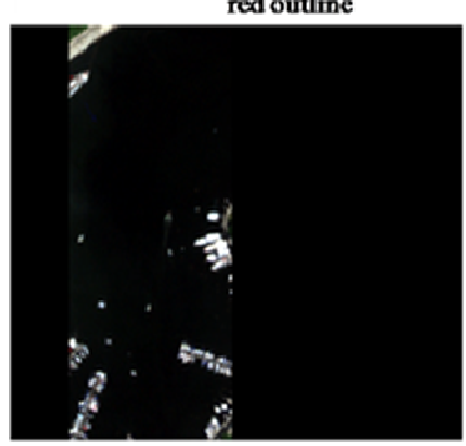

(d) Possiblesearch area within next frame

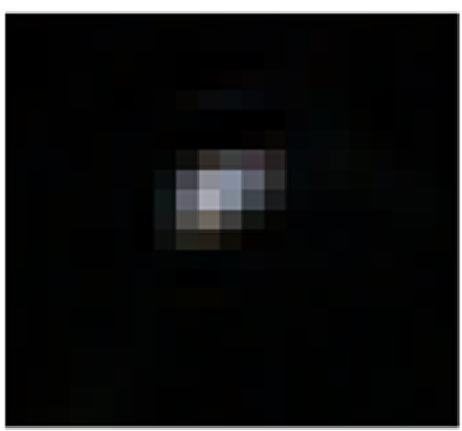

(b) Extracted ship target RGB image

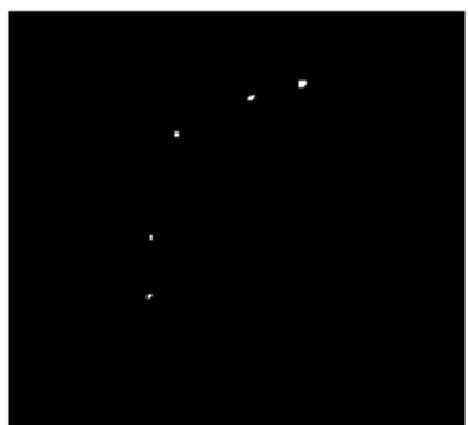

(e) Predicted trajectory of ship target

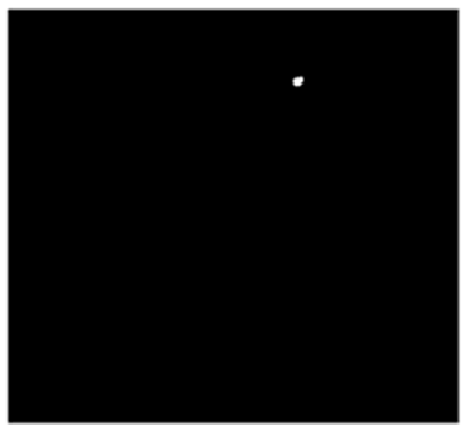

(c) Target binary image

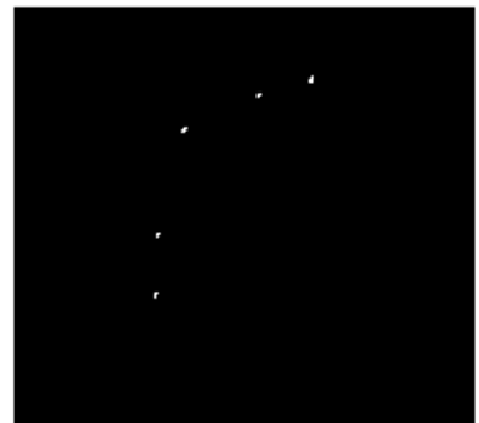

(f) Ground truth trajectory of ship target

Fig -5: Some intermediate results of proposed method for target tracking in harbor region database.

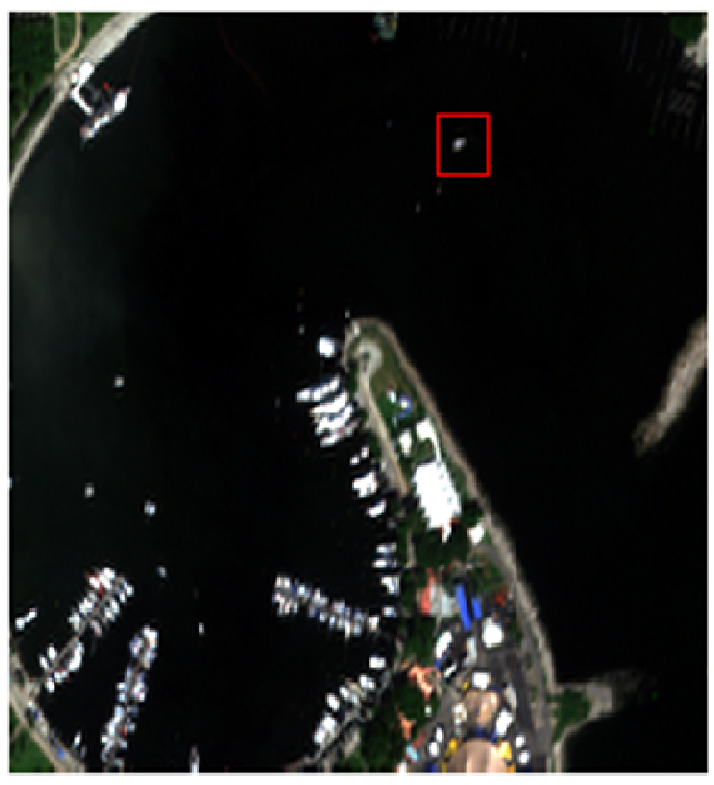

(a)

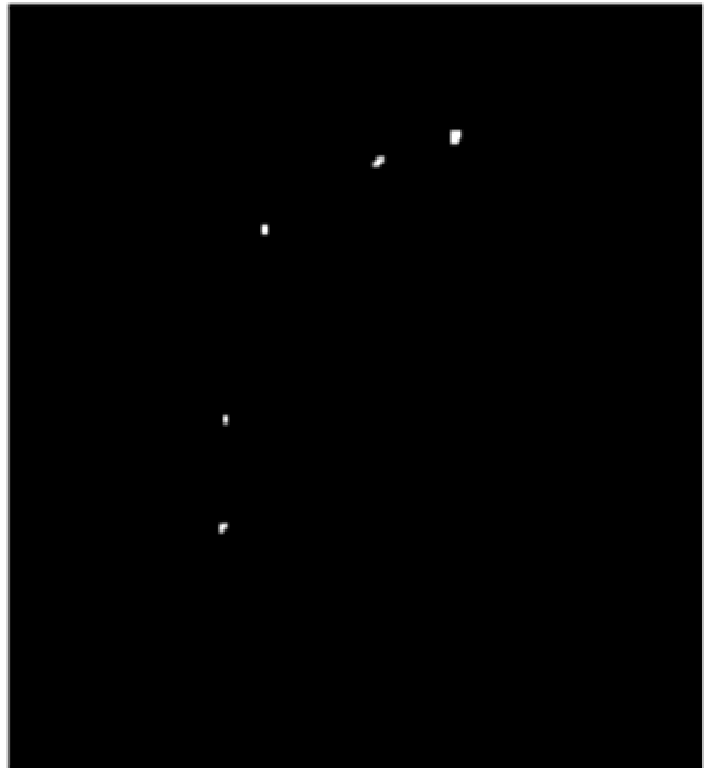

(b)

Fig -6: (a) Ship target in harbor region database within red outline (b) Predicted movement trajectory of ship target by proposed algorithm. 


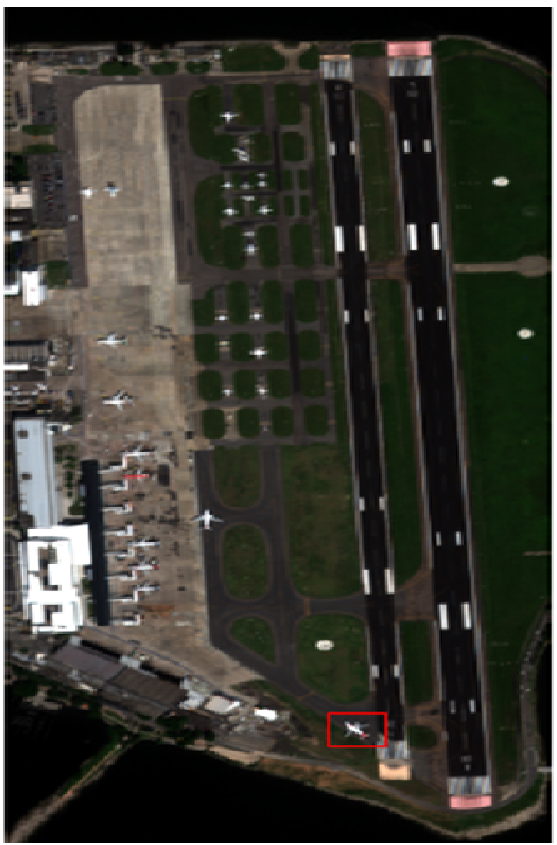

(a)

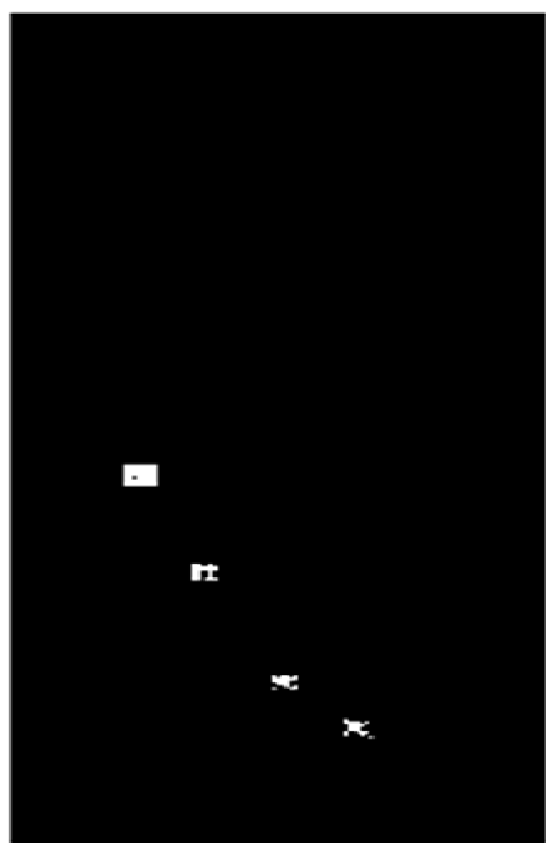

(b)

Fig -7: (a) Aircraft-1 target in an airport region database within red outline (b) Predicted movement trajectory of target aircraft-1 by proposed algorithm.

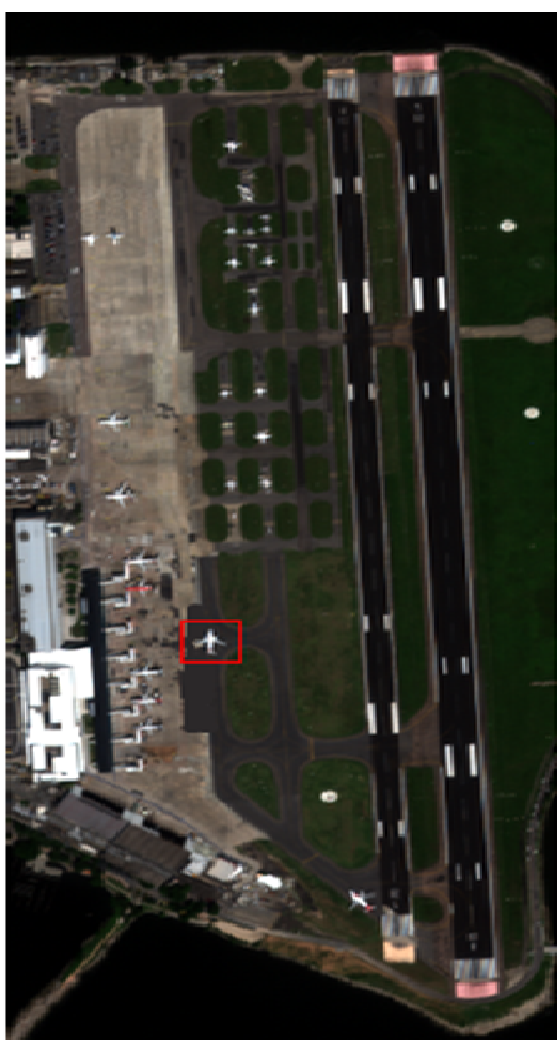

(a)

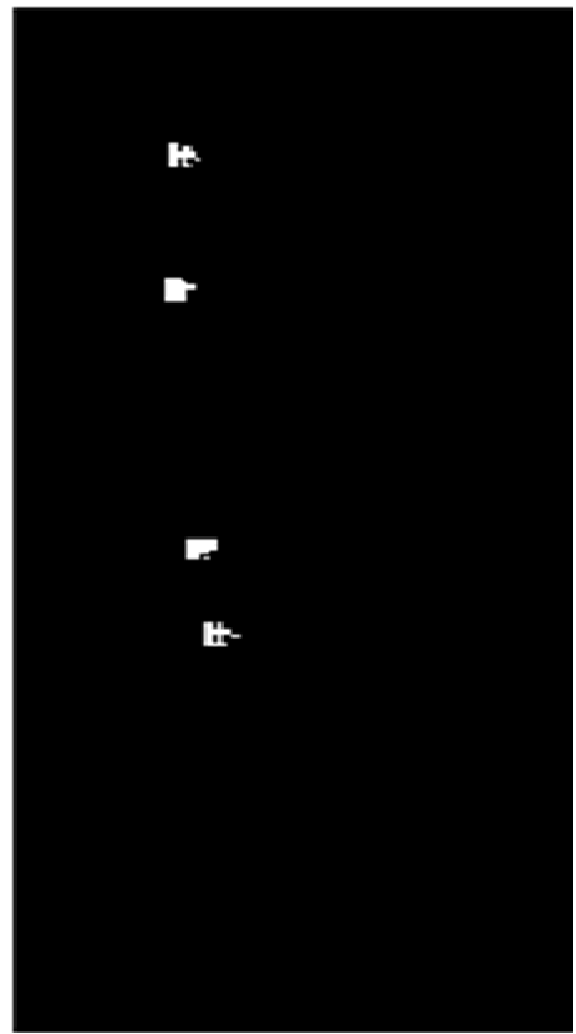

(b)

Fig -8: (a) Aircraft-2 target in an airport region database (b) Predicted movement trajectory of target aircraft-2 by proposed algorithm. 
Table -2: Comparison of tracking performance of Regional Operator Design (ROD) Based Algorithm [1] \& Proposed tracking algorithm with the help of some performance Parameters

\begin{tabular}{|l|c|c|c|c|c|c|}
\hline $\begin{array}{c}\text { Performance } \\
\text { Parameters }\end{array}$ & \multicolumn{2}{|c|}{$\begin{array}{c}\text { Regional Operator Design (ROD) } \\
\text { Based Algorithm [1] }\end{array}$} & \multicolumn{2}{c|}{ Proposed tracking Algorithm } \\
\hline & $\begin{array}{c}\text { Harbor } \\
\text { Region } \\
\text { database }\end{array}$ & \multicolumn{2}{|c|}{$\begin{array}{c}\text { Airport Region } \\
\text { database }\end{array}$} & $\begin{array}{c}\text { Harbor } \\
\text { Region } \\
\text { database }\end{array}$ & \multicolumn{2}{c|}{$\begin{array}{c}\text { Airport Region } \\
\text { database }\end{array}$} \\
\hline & Ship & Aircraft-1 & Aircraft-2 & Ship & Aircraft-1 & Aircraft-2 \\
\hline Recall & 0.690 & 0.833 & 0.925 & 1 & 0.949458 & 0.886503 \\
\hline Precision & 0.919 & 0.495 & 0.435 & 0.978260 & 0.894557 & 0.883792 \\
\hline F1 Score & 0.782 & 0.621 & 0.592 & 0.989010 & 0.921190 & 0.885145 \\
\hline Specificity & & & & 0.999985 & 0.999677 & 0.999604 \\
\hline Accuracy & & & & 0.999985 & 0.999533 & 0.999222 \\
\hline FDR & & & & 0.021739 & 0.105442 & 0.116207 \\
\hline
\end{tabular}
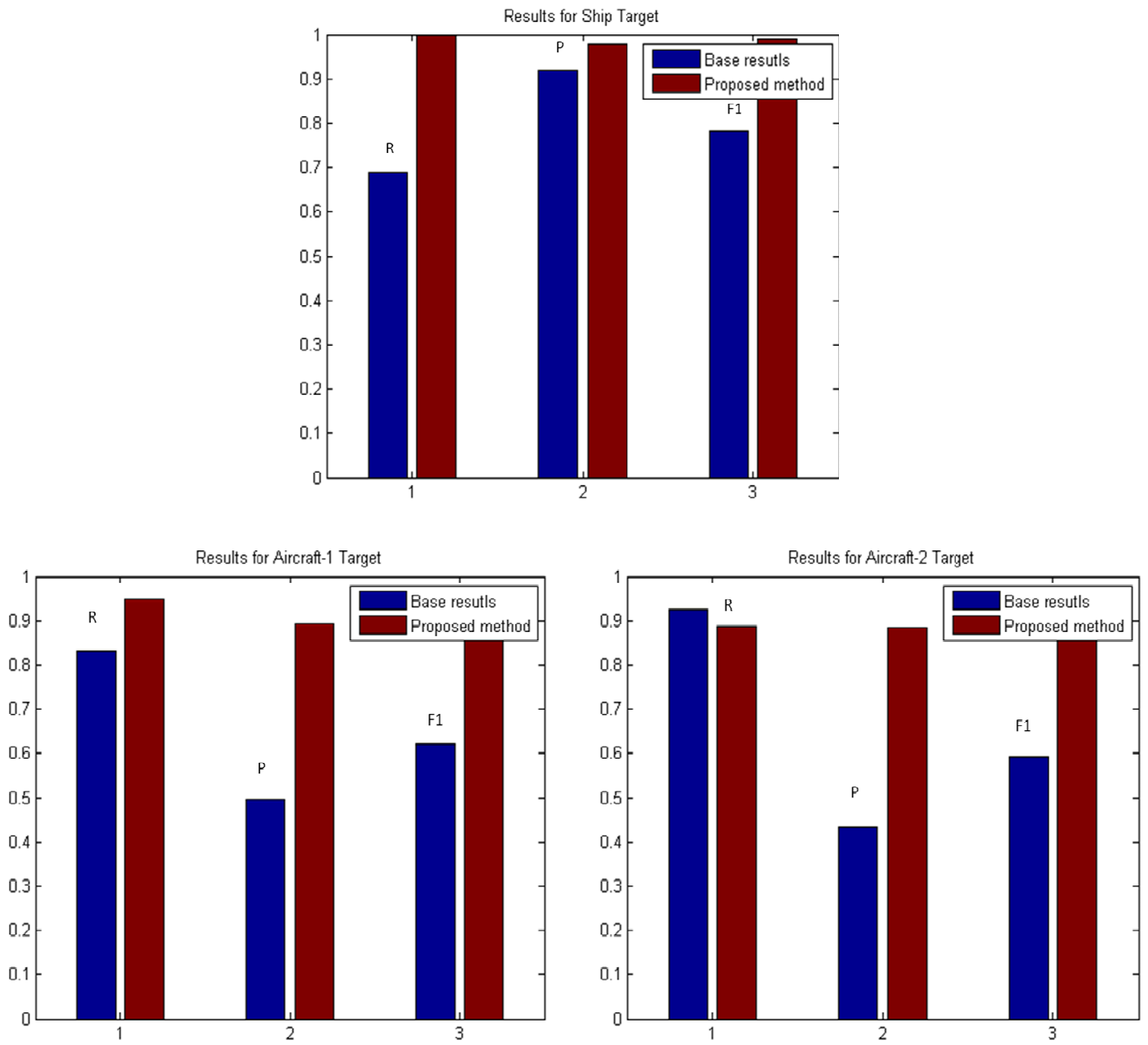

Fig -9: Graphical Representation of comparison results between Regional Operator Design based tracking algorithm \& Our Proposed Tracking Algorithm for three targets. 